\title{
OBPF: Opportunistic Beaconless Packet Forwarding Strategy for Vehicular Ad Hoc Networks
}

\author{
${ }^{1}$ Kashif Naseer Qureshi, ${ }^{1}$ Abdul Hanan Abdullah, ${ }^{2}$ Jaime Lloret and ${ }^{3}$ Ayman Altameem \\ ${ }^{1}$ Faculty of Computing, Universiti Teknologi Malaysia, Johor Bahru, Malaysia \\ [kashifnq@gmail.com, hanan@utm.my] \\ ${ }^{2}$ Universidad Politecnica de Valencia, \\ [jlloret@dcom.upv.es] \\ ${ }^{3}$ College of Applied Studies and Community Services, King Saud University (KSU), Riyadh, Saudi Arabia \\ [aaltameem@ksu.edu.sa] \\ *Corresponding author: Jaime Lloret
}

Received November 22, 2015; revised January 29, 2016; accepted February 10, 2016;

published May 31, 2016

\begin{abstract}
In a vehicular ad hoc network, the communication links are unsteady due to the rapidly changing topology, high mobility and traffic density in the urban environment. Most of the existing geographical routing protocols rely on the continuous transmission of beacon messages to update the neighbors' presence, leading to network congestion. Source-based approaches have been proven to be inefficient in the inherently unstable network. To this end, we propose an opportunistic beaconless packet forwarding approach based on a modified handshake mechanism for the urban vehicular environment. The protocol acts differently between intersections and at the intersection to find the next forwarder node toward the destination. The modified handshake mechanism contains link quality, forward progress and directional greedy metrics to determine the best relay node in the network. After designing the protocol, we compared its performance with existing routing protocols. The simulation results show the superior performance of the proposed protocol in terms of packet delay and data delivery ratio in realistic wireless channel conditions.
\end{abstract}

Keywords: Vehicular Network, Routing, Beaconless, Velocity, Simulation 


\section{Introduction}

The utilization of the vehicular ad hoc network (VANET) is envisioned for commercial and safety applications and is considered to be one of the most promising emerging fields of wireless technology in intelligent transportation systems. The main aim of vehicular communication is to assist vehicle drivers with real-time road information in order to avoid any critical danger by using vehicle-to-vehicle and vehicle-to-infrastructure architectures. Various mobile distributed applications are available to provide safety and comfort to travelers through a large range of traffic information dissemination: file-sharing and context-aware advertisement applications such as road safety, accident detection, traffic jam detection, weather information, multimedia and internet access. A large number of high-speed mobile nodes and frequently changing network topology leads to debate about how these applications can satisfy the delivery, delay and throughput challenges in the network [1-3]. Without effective multi-hop routing, these features are limited and several complexities and pitfalls exist in the network [4]. Therefore, an effective routing mechanism is needed for in-time data transmission in the network.

Geographical routing protocols are efficient approaches, where vehicles decouple packets from the node position and identify the route such as in greedy perimeter stateless routing [5], greedy perimeter coordinator routing [6] and vehicle-assisted data delivery [7]. These protocols consider the neighbors' and destination node positions obtained from location services (global positioning system (GPS) and Galileo positioning systems) and ensures progress towards the destination through flooding messages. The flooding messages (beacons) are periodically disseminated to inform the vehicle's one-hop neighbors about its presence and location [8]. The packet is forwarded through the next optimal neighbor relay node until the packet reaches the destination and each node keeps precise neighbor node information. Despite many advantages, these protocols have some issues. For example, these protocols do not perform well if they cannot find the next forwarding vehicle node, and therefore the network dynamics will increase [9]. If the node position information is not accurate, the selected vehicle node may not be the optimal hop and not in radio range. These factors lead to make inefficient and inaccurate forwarding decisions. The vehicle nodes need more accurate and up-to-date information through frequent beacon broadcasting [10]. Because of regular beaconing, the network will be congested and packet collision will occur, so the network may suffer communication overheads.

To address the frequent beaconing issues in the network, a number of beaconless forwarding approaches have been proposed such as contention-based forwarding (CBF) [11], beaconless routing protocol for vehicular environment (BRAVE) [12] and CoopGeo [13]. These beaconless approaches send a packet containing its own position and the destination's position in a data packet header and broadcast it to the next one-hop neighbor [14]. To overcome the limitations discussed above, we propose a beaconless geographical routing protocol with score functions that relies on a self-election scheme between vehicle nodes. The beaconless self-election forwarding is made in such a way as to maximize the network overheads and satisfy the quality of services in terms of the PDR and end-to-end delay in the network.

In the brief discussion provided above, it is clear that a trade-off between suitable metrics in network (e.g. vehicle directions and link quality) is essential for multi-hop routing. This paper proposes an opportunistic beaconless packet forwarding (OBPF) protocol for the VANET for packet forwarding. Protocol has two modes of operation and working differently, when 
candidate node is in between two intersection and when it is at the intersection. The proposed routing protocol is based on the forward progress, link quality and directional greedy approaches in order to improve packet delivery in the network. The proposed routing protocol has been simulated using the NS2 simulation tool. This protocol is suitable for different applications such as file sharing, chatting and other infotainment applications. The most important contributions of the protocol are as follows:

- Most routing protocols consider a single metric for data packet forwarding and do not work well in the harsh VANET environment. This paper proposes a multi-metric beaconless geographic routing protocol including most of the essential routing metrics such as forward progress, link quality and directional greedy forwarding.

- Wireless links are not stable in the VANET environment; to take this phenomenon into account, the proposed routing protocol considers the link quality during packet forwarding.

- We implemented the proposed OBPF protocol and compared it with existing beaconless and beacon-based routing protocols. The results showed the superiority of the proposed routing scheme as compared to LIAITHON, BRAVE and connectivity aware intersection based routing (CAIR).

The remainder of this paper is arranged as follows: Section 2 discusses the related literature. Section 3 provides a detailed description of the proposed strategy, including the flow chart and algorithm. In Section 4 , the simulation setup is presented. The performance evaluation of the proposed protocol is elaborated in section 5 , and we highlight the feasibility of the proposed protocol by considering a realistic city environment. Finally, last section presents our concluding remarks.

\section{Related Works}

In this section, we discuss and detail the previous proposed beacon and beaconless approaches for VANETs.

Chen et al. [15] proposed CAIR by selecting the best route with the help of the higher probability of connectivity. It is an intersection geographical-based protocol that works in three main phases: selection of intersection, prediction-based greedy forwarding, and recovery mode. The authors used the rectangle restricted area method for the first phase, where the positions of the source and destination node are formed with a line connecting them as an axis and the restricted area can be bound by an ellipse. The intersections are involved in a routed path which determines their position in the rectangle area. However, the traditional greedy forwarding strategy may lead to an error in the neighbor list and prevent some optimal relay nodes in the network. To addresses this issue, CAIR uses the position-based prediction algorithm based on velocity and location obtained from beacon messages. If the protocol faces the local maximum issue, the recovery mode is available based on the carry and forward approach. The city environment is congested with different types of obstacles, so the protocol faces a delay issue in the network because of its rectangle strategy.

Mohit et al. [16] proposed the guaranteed delivery beaconless forwarding scheme, where the next candidate node is selected through request-to-send/clear-to-send (RTS/CTS) control frames at the media access control (MAC) layer and the waiting time function to select the best next hop. The proposed scheme is based on greedy and recovery modes. In the greedy mode, 
the closest node to the destination responds first to the source node. In the case of the recovery mode, if the source node has the shortest route to the destination compared with the direct neighbor node, the contention winner node will be selected, which is near the source node. The source node establishes a link because of this other node's exit from the contention phase. The performance of the proposed scheme is better in terms of the PDR compared with other existing beaconless approaches. This is because the existing beaconless approaches retransmit the entire data, leading to duplicate packets and redundant retransmission issues. The guaranteed delivery beaconless forwarding scheme performance is better in terms of data delivery and low overheads at the MAC layer. However, the scheme is based on single criterion for measuring the waiting timeout, which is not enough for high-performance vehicular networks. The proposed scheme performs well in a highway environment and is not designed for the urban environment.

In another scheme [11, 17], the authors proposed a CBF scheme to select the next hop through the distributed contention process on the real-time location of present neighbors. This protocol does not maintain route, it works without a proactive strategy, where protocol maintains complete route such as in topology based routing. In the CBF scheme, the forwarding node sends control frames to neighbor nodes and the neighbor nodes take the decision of forwarding the packet. Then, the next forwarder relays the node selected through a distributed timer and based on self-election in the contention period and determines the relay node that has the shortest reply time and more geographical progress toward the destination. The selected node replies with the CTS frame to the source node and the other candidate nodes cancel and exit from the contention process when they hear the CTS frames. The forwarding node sends a complete message, which represents whether or not its neighbors shall forward the message. The authors tested this scheme with the beacon-based routing protocol, namely, greedy perimeter stateless routing, with the non-greedy forwarding disabled. The experiments were conducted with two different MAC standards (IEEE 802.11 and idealized MAC (0-MAC)). The CBF achieved a high PDR. The CBF considers movement, direction and power signal strength and neglects instability and unreliability issues in packet forwarding, which may lead to sub-optimal issues in wireless channels.

A new direction toward routing protocols was taken by [18] who proposed road-based routing with the help of a navigation system to establish a route between the source and destination. The authors used a sequence of intersections with high network connectivity for forwarding the packets toward the destination. Further, the authors eliminated the beacon packet and enhanced the receiver-based self-election to address the issue of network overheads. For beaconless forwarding, the authors used optimization between intersections for packet forwarding and did not consider the packet forwarding decision at intersections. In addition, they concentrated on three factors for packet forwarding (distance, optimal transmission range and power signal).

Rurup et al. [19] proposed another beaconless georouting scheme based on the self and protest principle with two methods for reactive face routing for packet delivery. The first method is the beaconless forwarder planarization to regulate the correct edge of the local planner subgraph without hearing from neighbors and the second method is angular relaying to determine the next hop of a right-hand traversal. Further, the authors used the circular neighborhood graph to solve the planarization issues of the Gabriel Graph because fewer messages are required to make the circular neighborhood graph than the Gabriel Graph. The experimental results showed that the function of distance and angle reduced the number of protest messages through a factor of 2 compared to the angle-based delay function.

In [20], an intelligent beaconless geographical routing algorithm was proposed to enable 
vehicles to forward packet along a city street efficiently. The protocol is based on reformed IEEE 802.11 RTS/CTS frames with the source and destination distance, signal strength and direction metrics. For stable and reliable packet forwarding, the candidate node considers the relative direction and power signal to elect itself intelligently. The simulation results showed that the intelligent beaconless protocol responded positively in terms of average delay and PDR in the urban vehicular scenario.

The authors in [21] proposed a location aware multipath video streaming scheme (LIAITHON) for the urban vehicular network based on location information for discovering the optimal route. It is a multipath receiver-based protocol for minimizing the collisions and congestion through a reduction in the packet length compared to the single path protocol. The degree of closeness and the selection of the forwarding zone are used for the relay node for transmitting the packets for the interval of reservation time toward the destination. The forwarding node selection is based on geographical advance, link stability and degree of closeness. The degree of closeness is responsible for discovering two relatively short paths with minimal route coupling effect. The protocol does not address the impact of distributing multiple video flows in the network, in the presence of more flow traversing and same communication range, where the coupling effects occur.

Cristiano et al. [22] proposed a resilient location-aware video unicast scheme (VIRTUS) which is a reactive receiving-based protocol for the selection policy of relaying nodes. The protocol uses the current and future locations with the help of three factors (forwarding zone definition, reservation time estimation and waiting time calculation) and with the help of Bayesian state estimation. The limitation of this protocol is the constant value of the forwarding zone directed toward the destination. It is not suitable for the urban environment because vehicle nodes are moving along the road.

Ruiz, et al. [23] proposed BRAVE based on spatial awareness and beaconless geographic forwarding. The spatial awareness refers to allowing the intermediate nodes to change the initial plan based on the view of the street map and local information. The trajectory of the packet is computed at every forwarding node and the next junction selection is based on Dijkstra's shortest path algorithm [24]. The protocol uses four types of messages (date, response, select and acknowledgement). The protocol uses the store and forward strategy instead of recovery mode. The protocol performance is better in terms of PDR and packet dropping in cases of high density. On the other hand, the less dense situation experiences high end-to-end delays and network overheads.

Ping et al. [25] proposed a beaconless geographic multipath routing protocol to construct maximum node disjoint multiple paths. In this protocol, each node selects multiple paths for forwarding the data packets within a disjointed subzone divided through a division algorithm. Before sending the packet, the source node calculates the distance toward the destination and calculates the subzones based on the number of acquired paths and the coefficient of each curve for the zone division. Then, the source node records the location information of itself, the destination and the coefficient of curves into a packet head and forwards the data packet to the next node. The forwarding strategy finds node-disjoint multiple paths in the network with high node density and maximizes node-disjoint multiple paths in the network without beaconing.

The comprehensive review of the literature illustrated that receiver self-election is significant for multi-hop routing for the city environment. To this end, we propose an OBPF strategy for the VANET environment. 


\section{Assumptions and Protocol Framework}

In this section, the complete overview of the beaconless approach is discussed, including its assumptions, forwarding process, flow diagram, algorithm and routing metrics.

To make our work possible, the following assumptions are made:

1. All vehicle nodes in our work are equipped with GPS and navigation systems to obtain their geographical position and speed information.

2. Vehicles are installed with a pre-loaded digital map for detailed road topologies such as own position, road segments and coordinates of the junctions.

3. For simplicity, the dead-end roads are not taken into account during the simulations.

\subsection{OBPF Protocol Description}

The proposed OBPF protocol is a source-based routing protocol capable of finding the robust route in the urban environment. The protocol is based on receiver self-election to suppress the effect of frequent hello or beacon messages, and next hop self-election is based on modified RTS/CTS frames of the IEEE 802.11 protocol. The IEEE 802.11 with the distributed coordination function [26] is designed to implement the Carrier sense multiple access with collision avoidance CSMA/CA protocol with RTS/CTS sessions. Whenever the vehicle source node wants to send the data, it senses the wireless channel for a specific time called the short inter-frame space. If the channel is suitable, then it selects a random back-off timer in the range of 0 , contention window. When the time is expired, the source vehicle node sends the RTS frame to the projected receiver and the receiver disseminates the CTS frame to its neighbors. The neighbors and the receiver node update the network allocation vector for a time interval and all the neighbors defer their transmission until the session is completed. When the source vehicle node receives the CTS frame, it forwards data to the receiver node after the acknowledgment (ACK) frame. In the case of transmission failure, the source nodes retransmit until the retry limit is reached.

OBPF is designed for inter-vehicle communication without fixed infrastructure and beacon messages for the vehicular urban environment. The protocol takes the packet forwarding decision between two intersections and at intersections to forward the packet onward to the destination. The source vehicle node tries to access the channel based on the multi-forwarding decision with link quality and forward progress metrics between the source and the candidate vehicle node. Before the source node transmits a data packet, it must determine its own location and include this information in the packet header. After broadcasting the modified RTS frame source, the node may wait during a score function value for its neighbors to decide about the transmission of data. The neighbors within the range of the source node know the source node position by analyzing the packet header. In addition, the source node is aware of its own position and destination position and determines its forwarding area.

At the intersection, the forwarder node broadcasts the modified RTS frame to all direct neighbors and, this time, the next forwarder is selected with directional greedy mode (DGM) and link quality metrics. When the best forwarder node that is at or between intersections accesses the channel, the remaining vehicle nodes cancel the frames. Whenever the source or carrier node receives a CTS frame from a neighbor node, then it transmits the data frames to the contention winner vehicle node after a short inter-frame space. The RTS frame modification is based on the score function and is used for the reply timer; it contains the position of the source and destination nodes with two flags. The first flag is used by the receiver to know about the source node position (i.e. whether it is at an intersection or between 
intersections) and the second flag deals with the respond/process of RTS messages. Fig. 1 shows the packet forwarding process between intersections (a) and at the intersection (b). In Fig. 1 (a), the source node sends RTS frame to A, B, C and D nodes and receive CTS reply from Node B, then node B start the same process to find next forwarder. In Fig. 1 (b), when the node is at the intersection again it sends RTS frames with different metrics to B.C and D and CTS reply receive from node $B$ and switch back to first mode of operation (between intersection) and then check next intersection. In last through node $\mathrm{H}$ protocol reaches to destination.
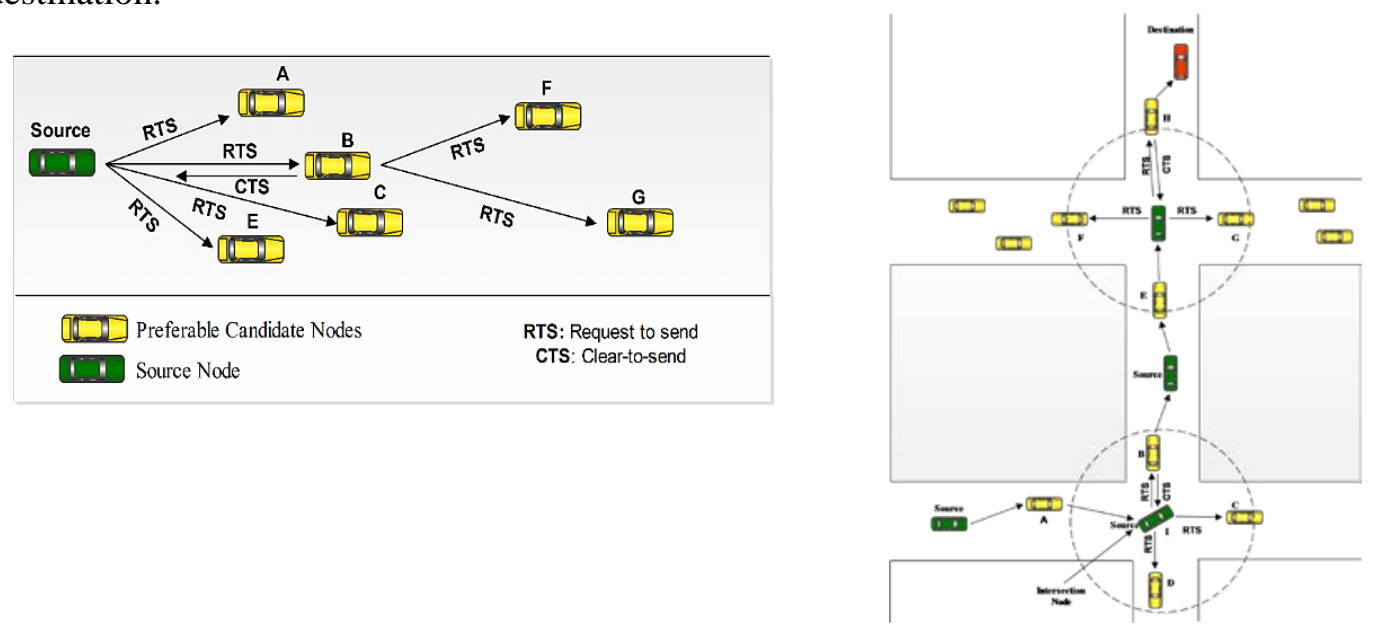

Fig. 1. Illustration of the protocol between intersections (a) and at the intersection (b).

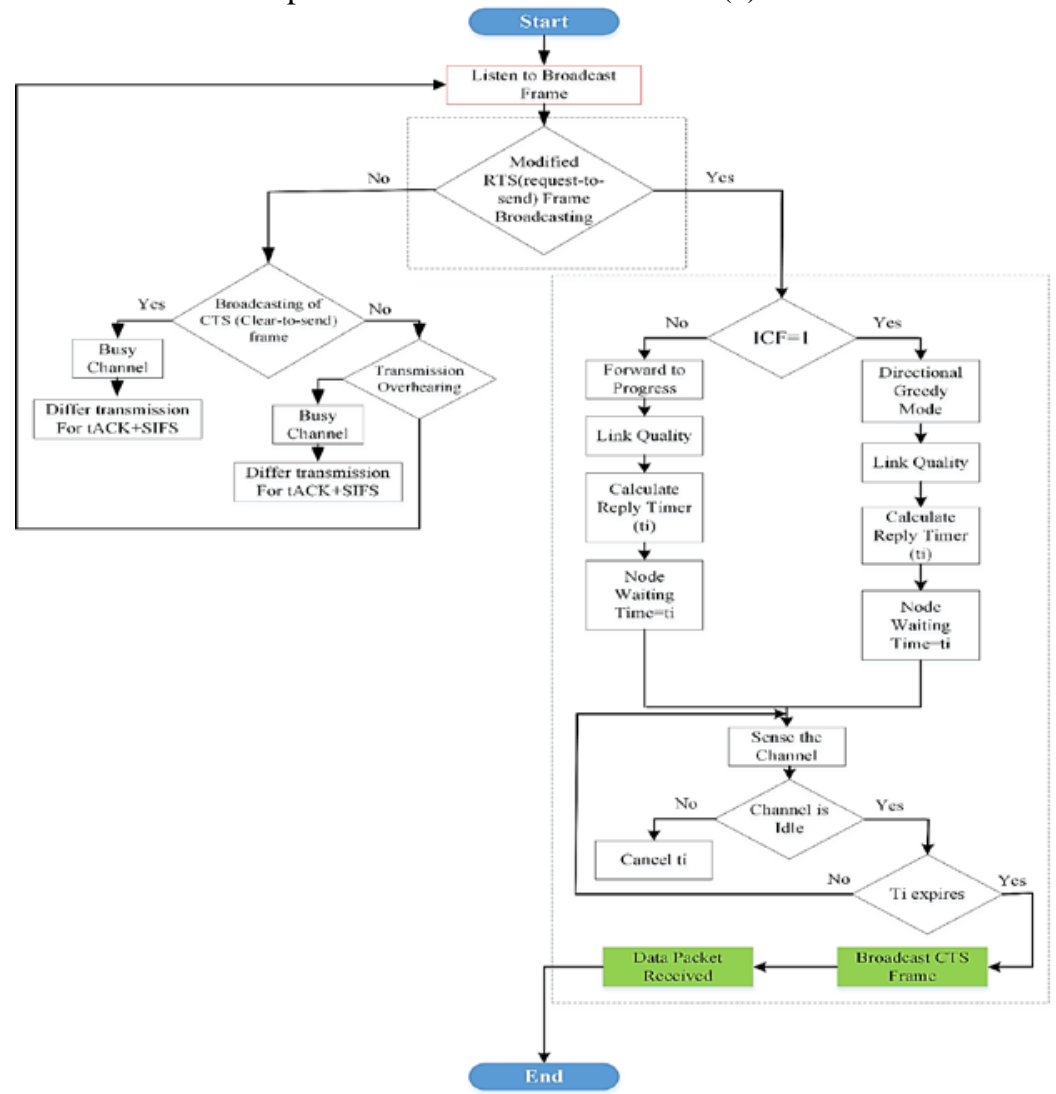

Fig. 2. Flow chart of OBPF Protocol 
Algorithm 1 shows the OBPF protocol function in detail. Lines 1 to 2 show that the source node sends the RTS frame to the candidate nodes with the intersection identifier flag to identify the position of the carrier node between intersections or at an intersection. If the flag value is 0 , then (Line 2) the candidate node calls the waiting function with the forward progress and link quality metrics and calculates the reply time between intersections. Then, the candidate nodes compute their reply timer ( $t i$ ) which depends on the link quality and forward progress (Lines 4 and 5). On the other hand, if the candidate node is at the intersection with flag value 1 , then candidate node select directional greedy forwarding and link quality (Lines 11 to 12). After determining the reply timer, the candidate node sets a value according to $t i$ (Lines 14 to 16). If the reply timer is finished, then a control CTS frame is transmitted from the carrier node which indicates its best relay node state. Meanwhile, other neighbor nodes cancel their timer when they hear the CTS frame. Then, the source node takes the decision to forward the data packet to the elected node (Lines 18 to 26).

If reply timer $t i$ has a negative value, the packet will be discarded from the candidate node side. The next section discusses the score function for the relay self-election between intersections and at intersections. List of main symbols used in OBPF algorithm with description are shown in Table 1.

Table 1. List of notations

\begin{tabular}{|l|l|}
\hline Symbol & Description \\
\hline $\mathrm{t}$ & Time to transmit data frame \\
\hline RTS & Receive-to-send \\
\hline CTS & Clear-to-send \\
\hline ACK & Acknowledgment \\
\hline SIFS & Short inter frame space \\
\hline $\mathrm{L}_{\mathrm{i}}$ & Location of node $\mathrm{n}_{\mathrm{i}}$ \\
\hline $\mathrm{L}_{\mathrm{d}}$ & Location of destination node \\
\hline $\mathrm{L}_{\mathrm{c}}$ & Location of packet career node \\
\hline $\mathrm{C}$ & Address of packet carrier node \\
\hline $\mathrm{IIF}$ & $\begin{array}{l}\text { Intersection identifier flag for identify the } \\
\text { position of carrier node between or at } \\
\text { intersection. }\end{array}$ \\
\hline $\mathrm{ti}$ & reply timer for node $\mathrm{n}_{\mathrm{i}}$ \\
\hline
\end{tabular}

Algorithm 1 Opportunistic beaconless packet forwarding at node $n_{i}$

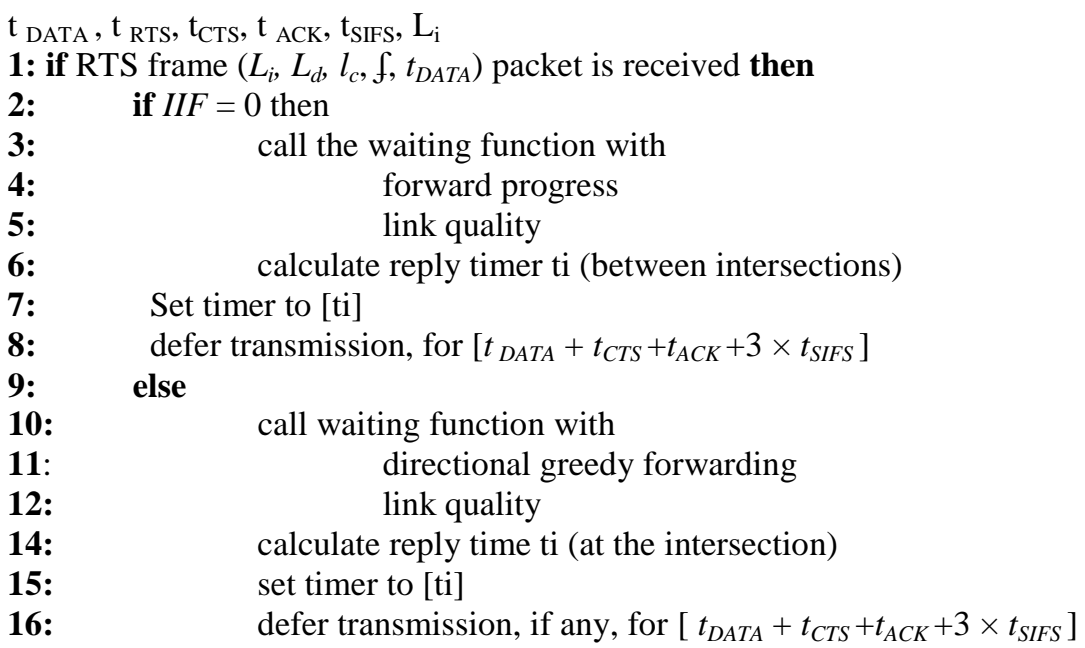




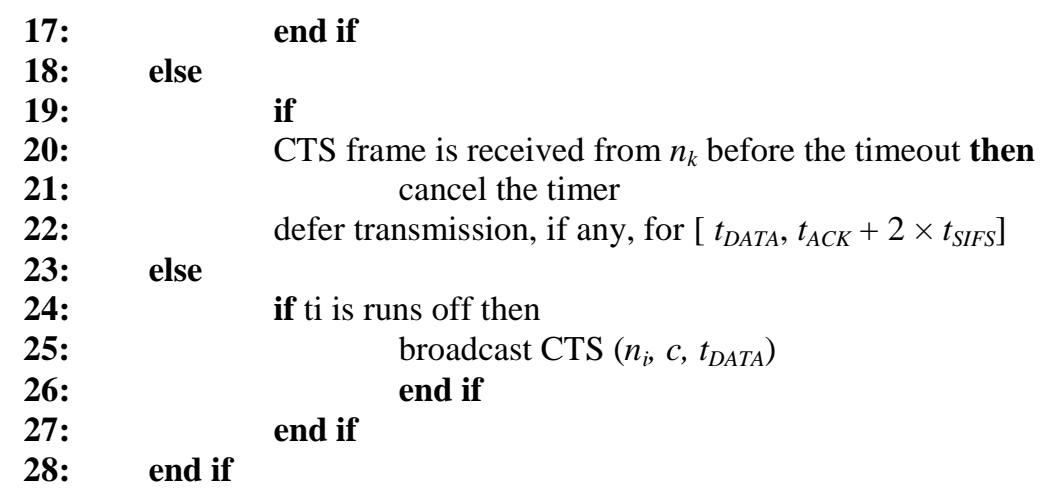

\subsection{Score Self-Election between Intersections}

The reply timer is based on a multimetric score function to select an optimal forwarder node. To qualify the forwarder node, we set some parameters including the link quality and the forward progress of the RTS frame between intersections.

\subsection{Forward Progress}

The forward progress metric is used for the geographical advance of the forwarder node toward the destination node with respect to the source node. The forwarder node FNi (a subset of forwarder nodes is considered as a relay node) is selected with high progress towards the destination node and computed as $\epsilon[0,1]$ according to Eq. (1):

$$
\text { Forward progress }= \begin{cases}\frac{2 R-P\left(F N_{i}, S\right)}{2 R} & \text { if } D\left(F N_{i, D}\right)>R \\ \text { if } D\left(F N_{i}, D\right)>R\end{cases}
$$

We denote $D\left(F N_{i} D\right) \in[0, \mathrm{R}]$ as the Euclidian between $F N_{i}$ (the subset of forwarder nodes considered as a relay node) and $D$ (destination node), $R$ as the radio range and $2 R$ as the maximum progress. The sum of two segments $\left(P_{1}\left(F N_{i}\right)+\mathrm{P}_{2}\left(F N_{i}\right)\right)$ composes the geographical advance $P(F N i, S) \epsilon[0,2 R]$ of a given $F N_{i}$ toward the destination node $D$. We define $P_{1}\left(F N_{i}\right) \epsilon$ $[0, R]$ as the projection of the distance travelled from $S$ to any $F N_{i}$. On the other hand, the projection of Line $F N_{i}-F N_{i}$ on Line $S$ - $D$ defines $P 2\left(R N_{i}\right) \in[0, R]$.

\subsection{Link Quality}

Through the link quality metric, we analyze the factors related to reliable transmission such as high packet delivery in the network. Existing beaconless routing protocols work on a circle transmission range and assume that nodes are within the transmission range. However, the vehicular environment is dynamic and the nature of the wireless links is asymmetric [27]. In this context, we consider link quality between two vehicle nodes as part of the DFD [28]. The DFD is a timer for forwarding decisions in the beaconless routing protocol, by using Eq. (2) in the interval $(0,1)$ :

$$
\text { Link Quality }=\left\{\begin{array}{lcc}
{ }^{1} L Q A_{M a x}-L Q A_{j} & \text { if } L Q A_{j}>L Q A_{\text {optimal }} \\
\frac{L Q A_{M a x}}{L} & \text { if } L Q A_{\text {Worst }}<L Q A_{j}<L Q A_{\text {Optimal }} & \text { (2) } \\
0 & \text { if } L Q A_{j}<L Q A_{\text {Worst }} & \text { are }
\end{array}\right.
$$


classified according to the values of the packet reception ratio (PRR) into three regions of connectivity with different percentage ranges such as connected (PRR $>90 \%$ ), transitional (PRR between $10 \%$ and 90\%) and disconnected (PRR $<10 \%$ ). In this context, we present the bounds of disconnected and connected regions by means of two $L Q A$ thresholds: $L Q A_{\text {Optimal }}$ and $L Q A_{\text {Worst }}$. Based on these thresholds, we can classify a link $e_{j}$ as disconnected, when receiver vehicle node $R V_{i}$ receives a packet with $L Q A_{j}$ and lower than $L Q A_{\text {Worst }}$; or, we can classify a link as connected when $L Q A_{j}$ is higher than $L Q A_{\text {Optimal }}$; or, we can classify a link as transitional for $L Q A_{j}$ ranging between $L Q A_{\text {optimal }}$ and $L Q A_{\text {Worst }}$.

According to Eq. (1), FNi (the subset of forwarder nodes considered as a relay node) with a connected link to $S$ (source node) has a higher probability of forwarding the packet faster (link quality $=0$ ), and is considered to have high reliability in the network. For disconnected links, link quality returns 1 which is considered to be low quality for forwarding the packet. The transitional link generates values ranging from 0 to 1 , indicating unreliable link quality.

Now we describe the score function trade-off between the forward progress and link quality, which is given by:

$$
g\left(F P_{i}, L Q A_{i}\right)=A \times F P_{i}^{\alpha_{1}} \times L Q A_{i}^{\alpha_{2}}+B_{\max }
$$

where $\alpha_{1}$ and $\alpha_{2}$ are weights for FP and LQA routing metrics and variable $B_{\max }$ denotes the maximum time delay after receiving the RTS frame. A is defined as follows:

$$
A=\frac{-B_{\max }}{F P_{\max }^{\alpha_{1}} \times L Q A_{\max }^{\alpha_{2}}}
$$

To compute the score function in Eq. (3), it is essential to find the maximum values of $F P_{i}$ and $L Q A_{i}$, where the $F P$ value depends on the simulation setting such as the area and communication range. On the other hand, the maximum value of link quality $L Q$ is set to 0 for higher probability of forwarding the packet faster.

\subsection{Score Self-Election at an Intersection}

At the intersection, the optimal forwarder node is determined based on the reply timer multimetric score function. The reply timer is based on three input parameters: predictive mode, link quality, and recovery mode.

\subsection{Directional Greedy Mode}

When the forwarder node is at the intersection and receives the RTS frame, it switches to DGM and selects the next forwarder node, which is closer to the destination. The simple greedy forwarding leads to the routing loop because of multi-directional vehicle movements and increases the end-to-end delays in the network. We add direction and node position metrics in greedy forwarding to select the next hop moving toward the destination node. If only direction is used in greedy forwarding, it will cause more hops with delays in the network. The next hop selection is possible through the weighted score function calculation as follows:

$$
\text { weighted Score } \text { S }_{i}=\alpha(1-S D i / F D c)+\beta \cos \left(\overrightarrow{v_{i}}, \overrightarrow{p_{i}},{ }_{d}\right)
$$


$\alpha$ and $\beta$ denote the weight position and direction factors and are calculated as $\alpha+\beta=1$; where $S D_{i}$ represents the shorter distance from intersection node $i$ to the destination node and FDc shows the shortest distance from the forwarder node to the destination node. SDi/FDc shows the closeness of the next candidate hop, $\overrightarrow{v_{i}}$ shows the vector for vehicle speed, $i ; \overrightarrow{p_{i, d}}$ shows the vector from the location of node $i$ to the location of the destination node, and $\left.\cos \overrightarrow{\left(v_{i}\right.}, \overrightarrow{p_{i, d}}\right)$ denotes the cosine value for the angle made by these two vectors. The cosine value is used to differentiate the priority of vehicles according to the vehicles' direction of movement. The value of Weighted Score ${ }_{i}$ is used to select a next forwarding hop or the value of Weighted Score $_{i}$ is used to select a next forwarding hop, and the high value of the weighted score in the neighbor nodes is used to select the next forwarder.

\subsection{Link Quality}

The urban environment is susceptible to fading and attenuation due to different obstacles including trees and buildings. Therefore, link quality metric is suitable here to deal with fading and attenuation issues. The candidate node can determine the link quality parameter in the RTS frame.

After all the routing metrics have been defined, there is a need to combine these criteria into one function. We used an aggregation function to make the score function into one single ranking measure. The random back-off timer of the IEEE 802.11 standard is based on a slot timer with a random number, and the score function is also based on the multiplication of specified variables. The score function is based on $\mathrm{L}$ routing metrics $\mu_{\mathrm{i}}\left\{\mu_{\mathrm{i} 1}, \mu_{\mathrm{i} 2} \ldots \ldots . . \mu_{\mathrm{iL}}\right\}$ and, for each of them, candidate node $n_{i}$ has a numerical value in the range of $\left[u_{i}^{\text {Min }}, \mu^{\text {Max }}\right]$. For our proposed solution, we assume that the routing metrics are maximized values. The multimetric function is presented as follows [29]:

$$
h\left(\mu_{i 1}, \mu_{i 2} \ldots \ldots \ldots . . . \mu_{i 1}\right)=Y \times \mu_{i 1}^{\gamma_{1}}, \mu_{i 2}^{\gamma_{2}}, \ldots \ldots \ldots . . . \mu_{i L}^{\gamma L}+X_{\max }
$$

where $\mathrm{X}$ denotes the maximum value of the scoring function and $\mathrm{h}\left(\mu_{\mathrm{i} 1}, \mu_{\mathrm{i} 2} \ldots \ldots . . \mu_{\mathrm{iL}}\right)$, $\mathrm{Y}$ is the variable dependent weights of the limiting condition, and $\left(\gamma_{1}, \gamma_{2} \ldots \ldots \gamma_{\mathrm{L}}\right)$ is an $L$ weight array used for giving priority to routing decision metrics. If the routing metric has a high weight factor, then it has more impact in the self-election process. In our OBPF protocol, we use two factors for packet forwarding at the intersection and the reply time value is calculated as follows:

$$
H\left(D G M_{i}, L Q_{i}\right)=Y \times D G M_{i}^{\gamma_{1}} \times L Q_{i}^{\gamma_{2}}+X_{\max }
$$

The maximum value of $H\left(D G M_{i}, L Q_{i}\right)$ is set when its derivative equals to zero, and the value of $\mathrm{Y}$ is given as follows:

$$
Y=\frac{-X_{\max }}{D G M_{\max }^{\gamma_{1}} \times L Q_{\max }^{\gamma_{2}}}
$$

To estimate the score function in Eq. (7) we need to evaluate the maximum values of $D G M_{i}$ and $L Q_{i}$, where the DGM value depends on the simulation setting such as the area and 
communication range. Accordingly the $D G M_{\max }$, the value is equal to (8). On the other hand, the maximum value of link quality $L Q$ is set to 0 for higher probability of forwarding the packet faster.

The maximum delay time $\left(\mathrm{X}_{\max }\right)$ for the candidate node election is $0.8 \mathrm{~ms}$, which is set in the simulation scenario, $\gamma_{1}=0.5, \gamma_{2}=0.3$; then, we calculate the value of Y with the help of Eq. (8), and then we calculate the reply timer $\left(t_{i}\right)$ with the help of Eq. (7), which is equal to $0.0840 \mathrm{~ms}$. This time is the waiting time of a candidate node when it receives the RTS frame. When the source node sends the RTS frame, all the neighbor nodes calculate this score function.

The basic purpose of the score function is to determine a single value with the help of different parameters in the protocol. The final decision is based on the final value of the score function.

\section{Numerical Results}

This section is divided into two parts: the simulation scenario, and emulation of the OBPF protocol. The first part introduces the complete simulation setup and related parameters in detail. The second part gives the outcomes of the simulations for evaluating the proposed protocol performance.

\subsection{Simulation Scenario}

The performance of OBPF was analyzed using the popular NS-2.34 network simulator with the model mobility generator for vehicular networks (MOVE). The mobility generator is used for realistic vehicular movement generation in the urban environment. MOVE is based on the simulation of urban mobility (SUMO). It is an open source micro-traffic simulator [30]. MOVE has two modules for building a vehicular environment, namely, the vehicle movement editor and the road map editor. The road map editor gives essential features of roads such as the number of lanes, roads, junctions and the traffic light setup. The vehicle movement editor is used to set factors such as the speed of vehicles, the number of vehicles and the probability of right or left turning. To set all the required parameters in the two editors, the trace file is generated by MOVE and directly used in NS-2. A satellite image of Kuala Lumpur, Malaysia, is obtained through Google Earth software and imported in ArcGIS [31] to build the map with up-to-date data and set road coordinates (Fig. 4). Then, the map is input in MOVE to incorporate further information in the map. Afterward, the trace files and other configurations are generated to analyze the OBPF protocol.

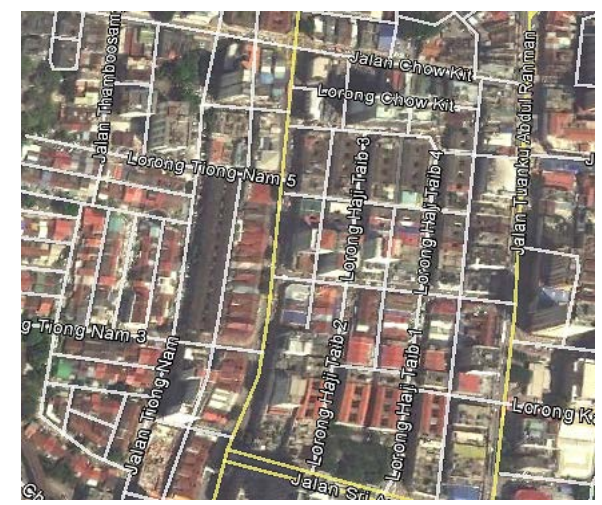

Fig. 4. City road map of Kuala Lumpur, Malaysia 
Furthermore, the shadowing model was used at the physical layer to characterize the wireless channel with the value of the path loss exponent $n=2.8$ and reference distance set at $\mathrm{d} 0=0.4$ [32, 33]. In addition, the communication range was set at $250 \mathrm{~m}$ and the number of vehicles nodes was between 100 and 250 with an average velocity from 25 to $50 \mathrm{~km} / \mathrm{h}$. The simulation parameters of the MAC layer were configured to meet the specifications of the IEEE 802.11 standard. The simulation parameters, summarized in Table 2, were based on realistic measurements between nearby vehicles [34]. To avoid the effects of transient behavior in the results, we set the settling time of 30 seconds in the simulation. For accurate simulation results, 25 simulations (on average) were run for each metric.

Table 2. Simulation Parameter

\begin{tabular}{|l|l|}
\hline Parameters & Value \\
\hline Simulation Area & $2500 \mathrm{~m} \times 1500 \mathrm{~m}$ \\
\hline Simulation Time & $350 \mathrm{~s}$ \\
\hline Number of Vehicles nodes & 100 to 250 \\
\hline Vehicle velocity & $25-50 \mathrm{~km} / \mathrm{h}$ \\
\hline Transmission range & $250 \mathrm{~m}$ \\
\hline Mac Protocol & IEEE $802.11 \mathrm{p}$ DCF \\
\hline Data Packet size & 512 bytes \\
\hline Channel Bandwidth & 3 Mbps \\
\hline Maximum packet generation rate & $0.5-5$ packets /second \\
\hline Weighting Factors $\alpha_{1}, \alpha_{2} \gamma_{1}, \gamma_{2}$, & $(0.01,0.01,0.2,0.2)$ \\
\hline
\end{tabular}

\subsection{Evaluation Methodology}

To evaluate the performance of OBPF protocol, we compared it with two well known beaconless geographical routing protocols, named BRAVE [12] and LIAITHON [21], and one well known beacon-based routing protocol, named CAIR [15]. BRAVE is a beaconless routing protocol based on spatial awareness to allow intermediate nodes to change the initial plan based on the street map and local information. This protocol is not efficient in low dense areas and leads to high end-to-end delays and network overheads. LIAITHON is a multipath receiver-based protocol which uses location information to discover the optimal route. It uses geographical advance, link quality and degree of closeness to forward the data toward the destination. CAIR is based on the high probability of connectivity. It is an intersection-based protocol with three operations: selection of intersection, prediction-based greedy forwarding, and recovery mode. For the first operation, a rectangle restricted area searching method is used to find the route in lrge-scale VANETs. The restricted area can be plotted by bounding the ellipse. The city environment is congested with different types of obstacles, so the protocol faces a delay issue in the network because of its rectangle strategy.

The routing metrics packet delivery ratio, end-to-end delay and average path length were used to compare the proposed OBPF protocol with the three state-of-the-art routing protocols. PDR measures the average number of successfully delivered data packets from the source to the destination. This metric shows the network ability of the routing protocol. The end-to-end delay metric measures the total time it took a packet to travel from the source to the destination node in the network. It is the sum of the processing delay, medium access, sending the buffer, relay election, retransmission and propagation delay. The average path length metric measures the average number of relay nodes that participate in forwarding the packet from the source to the destination node.

To test these metrics, we conducted various experiments with different parameters such as an 
environment with obstacles and an environment without obstacles, with different vehicle velocities and with different traffic densities in the urban environment.

\section{Results and Analysis}

In this section, we discuss the results from the various experiments conducted to analyze the protocol's performance.

\subsection{Effect of weighting factors}

We set different values in the simulation for weighting factors in order to determine the optimal balance values between routing metrics. Figures 6 (a) and (b) show the measured PDR and end-to-end delay versus packet sending rate with different weighting factors. First, we set the weighting metric values at $0.01,0.01,0.02,0.02$; initially, the deviation value of the source packet increased and the PDR and average delay were stable in the network. Whenever the packet generation rate reached $32 \mathrm{kbps} \mathrm{ms}$, the PDR slightly decreased by $750 \mathrm{~ms}$. In this case, the network saturation was at peak level (32 kbps is the maximum limit in stable situations). After $32 \mathrm{kbps}$, packet loss starts to occur in the network and causes the network load. Whenever weighting factors values are set with $0,0.1,0,0.02$, and only considered one one metric at or between intersections the PDR dropped compared to the first results. Again, the third and fourth time we changed the weighting factor values and observed that the PDR decreased constantly. Finally, the first analysis showed that when the weighting factor values were the same as each other in both scenarios, then the PDR was better compared to when the weighting factors had different values.

We tested the same values in terms of average packet delay in the network as shown in Fig. 6 (b), and observed that, when the weighting factor values were the same as each other, the data packets were successfully delivered with low delays in the network. The reason is that, if the factor values are different from each other, the OBPF protocol uses one factor in between the intersections and one factor at the intersection and does not show better performance. The analysis showed that using only one factor was not enough in the "between intersections" environment and the "at the intersection" environment for finding the shortest and optimal routing path in the network. The OBPF protocol supports and favors the following factors: forward progress and link quality (between intersections) and DGM and link quality (at the intersection). The weighting factors have an important impact in every election round because of the optimal reply timer of the contention phase between the candidate nodes.
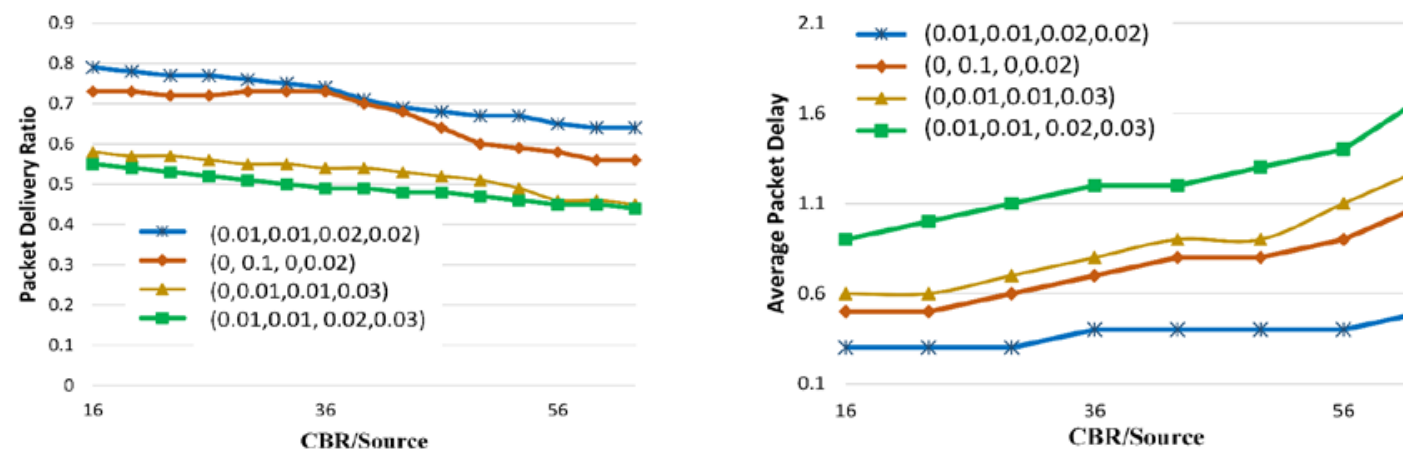

Fig. 6. Effect of different values of weighting factors in terms of

(a) PDR and (b) average packet delay 


\subsection{Effect of vehicle velocity}

For this experiment, the total number of vehicles nodes was 250, with 8 of them set as source nodes in the network. To determine the impact of vehicle velocity, we set the vehicle velocity at between $25 \mathrm{~km} / \mathrm{h}$ and $50 \mathrm{~km} / \mathrm{h}$ in an urban environment and we set the beacon interval at 0.5 ms for the BRAVE, LIAITHON and CAIR protocols. Fig. 7 presents the PDR of the OBPF protocol and the BRAVE, LIAITHON and CAIR protocols in terms of different vehicle velocities. Through these experiments, we determined that if the vehicle speed increased, the successful PDR decreased. However, the OBPF protocol performed better at high speed because of the use of the RTS frames instead of beacon messages for updating the neighbor information in the network. The beacon messages take more bandwidth compared to the RTS frames and lead to network overheads. The beaconless approach consumes less bandwidth and the percentage of link utilization will enhance the packet transfer rate. Multimetric-based election is favorable for making an optimal route between the source and destination. We observed that the OBPF protocol performance always lagged behind LIAITHON. This is because both protocols use the forward progress and link quality for forwarding the packets, but the OBPF performance is better because it uses DGM instead of the multipath strategy. Whenever the vehicles velocity at $50 \mathrm{~km} / \mathrm{h}$, the BRAVE and LIAITHON protocols dropped packets around $70.3 \%$. Then, we calculated the mean of these three protocols through analysis of variance in Excel. The results showed that the OBPF had lower variance than BRAVE and LIAITHON. The OBPF protocol is more reliable in urban environments to deal with high mobility and obstacles environment.

On the other hand, the CAIR protocol uses beacon messages to update the possible information about its neighbor nodes. These periodic beacon messages are stateless because of the high mobility of the vehicle nodes in the network. The CAIR protocol dropped $50 \%$ to $55 \%$ packets when the vehicles' speed was set at $50 \mathrm{~km} / \mathrm{h}$ in the network. The OBPF, BRAVE and LIAITHON protocols suffered less packet dropping compared to CAIR. The proposed protocol showed better results and one of the main reasons for this efficiency is the beaconless approach in the protocol compared with the other beacon-oriented protocol.

Fig. 7 (b) shows the average packet delay in terms of vehicle velocity. The proposed protocol had the smallest delay compared to the other three protocols. The modified RTS/CTS frames are used in the proposed protocol to determine an optimal route in the network and this method is more reliable and effective in reducing the traffic load on the MAC layer and leads to improved delays in the network. Through the modified handshake mechanism, less transmission and exponential back-off occur in the MAC layer. The CAIR protocol average packet delay increased drastically with high velocity because of the predictive nature of the protocol at the intersection and the beacon message broadcasting. In contrast, the BRAVE and LIAITHON protocols also suffered from average packet delay compared to the OBPF protocol. This can be attributed to the fact of beacon messages being used to update neighbor information and the relay node contending the access channel based on improved greedy forwarding where one transmission advance metric is used and the relay node selects itself as a next packet forwarder. The OBPF protocol uses the directional greedy approach in the RTS frame; if protoocl uses only greedy factor for RTS frames, it may leads to data packet losses due to unreliable wireless channels. Further it also causes of end-to-end delay due to redundant retransmission at MAC layer. The direction used in the OBPF protocol with the greedy approach at the intersection resolved the issue of redundant retransmission in the network. In addition, we compared the OBPF protocol with the three protocols in terms of hop count in the network to reach the packet to the destination (Fig. 7 (c)). The proposed protocol path was slightly longer than in LIAITHON because we used the metrics of link quality, forward 
progress and directional greedy forwarding. In contrast, the proposed protocol had shorter paths' compared to BRAVE because of its spatial awareness. The OBPF protocol performed better with shorter path lengths than BRAVE and CAIR and slightly longer than the LIAITHON protocol.
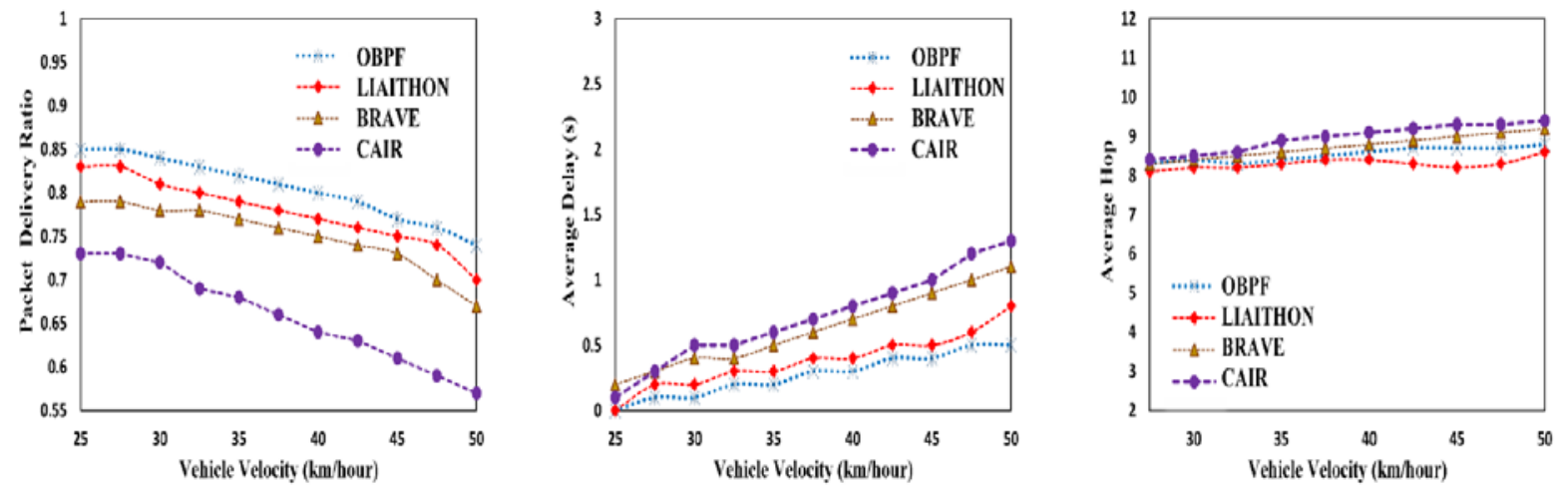

Fig. 7. Effect of varying vehicle velocity in the OBPF protocol compared with LIAITHON, BRAVE and CAIR routing protocols; from left (a) PDR, (b) average delay, (c) average hop

\subsection{Effect of different obstacles in the network}

We conducted experiments in the presence of radio obstacles and without obstacles in an urban environment in order to evaluate the proposed OBPF protocol's performance. We set the vehicle speed at $30 \mathrm{~km} / \mathrm{h}$ and set the traffic density at 150 vehicles, with 8 of them as source nodes in the network. We ran the simulation and set different packet generation rates and set building obstacles through the mobility generation model. Some streets were set without obstacles in order to interface with the radio signal, and street numbers were set in the road segment file. The packets are captured in the presence or obstacles and without obstacles for comparison. We also modified the attenuation value between the trimester and receiver. As shown in Fig. 8, the proposed OBPF routing protocol had better performance and increased up to $8 \%$ compared with the other protocols. One of the main reasons behind these results is the use of the beaconless approach and link quality instead of using the transmission range to select the next relay node in the network. On the other hand, Fig. 8 (b) shows the average packet delay plotted with respect to packet generation rate of protocols. Whenever the arrival time of the inter-packet was large, the average packet delay increased and was different with each protocol. The OBPF protocol average delay was about $520 \mathrm{~ms}$ and fluctuated between 520 and $700 \mathrm{~ms}$ at $72 \mathrm{kbps}$. When the vehicles were in the streets without obstacles, the average delay time was short and in the presence of obstacles it was high. The performance of the OBPF protocol was better than the performance of the other protocols. 


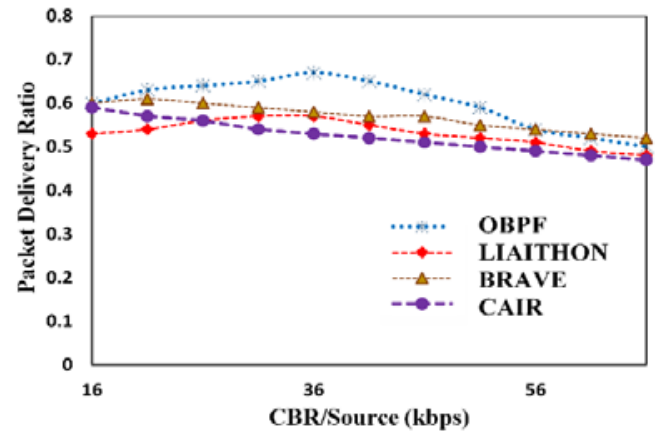

(a)

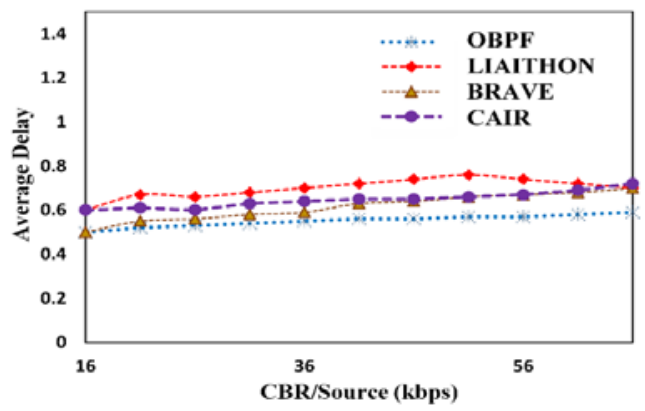

(b)

Fig. 8. Effect of radio obstacles on the performance of the OBPF protocol compared with LIAITHON, BRAVE and CAIR routing protocols; (a) PDR, (b) average delay

\subsection{Effect of traffic density}

In this section, we present the results on the effects of traffic density on the proposed routing protocol compared with the existing protocols. For the experiment, we ran a simulation with different vehicle speeds and different numbers of vehicles. In the first experiment, we set the vehicle speed at $40 \mathrm{~km} / \mathrm{h}$ and the vehicle node setting was between 150 and 250. Fig. 9 (a) shows the average delivery ratio. Whenever we increased the density (number of vehicles), the PDR consistently increased. This is not surprising because the probability of connectivity is increased with traffic density in the network. When the traffic density was set at 250, the proposed protocol trend was flat because of the RTS/CTS mechanism. The trends for the LIAITHON and BRAVE protocols were weak due to the spatial awareness in the network. The CAIR protocol uses prediction-based greedy forwarding and recovery mode, and this probably leads to link failure because of high signal attenuation in wireless channels. In this case, the OBPF protocol's performance is better.

The second metric is the average packet delay. As depicted in Fig. 9 (b), we observed that the average packet delay of the OBPF protocol steadily decreased when the number of vehicles was set at 200 and rose slightly when the number of vehicles was set at 250 . One of the main reasons behind these results is that high traffic density leads to packet duplication and collisions in the network. The average packet delay of the other two protocols increased in the presence of 250 vehicle nodes because of high latency at the MAC layer retransmission.

Fig. 9 (c) shows the average path length variation with high traffic density in the network. The OBPF protocol hop count was slightly longer compared to LIATHON, BRAVE and CAIR. However, the proposed protocol offered better performance in terms of successful delivery ratio and PDR. 

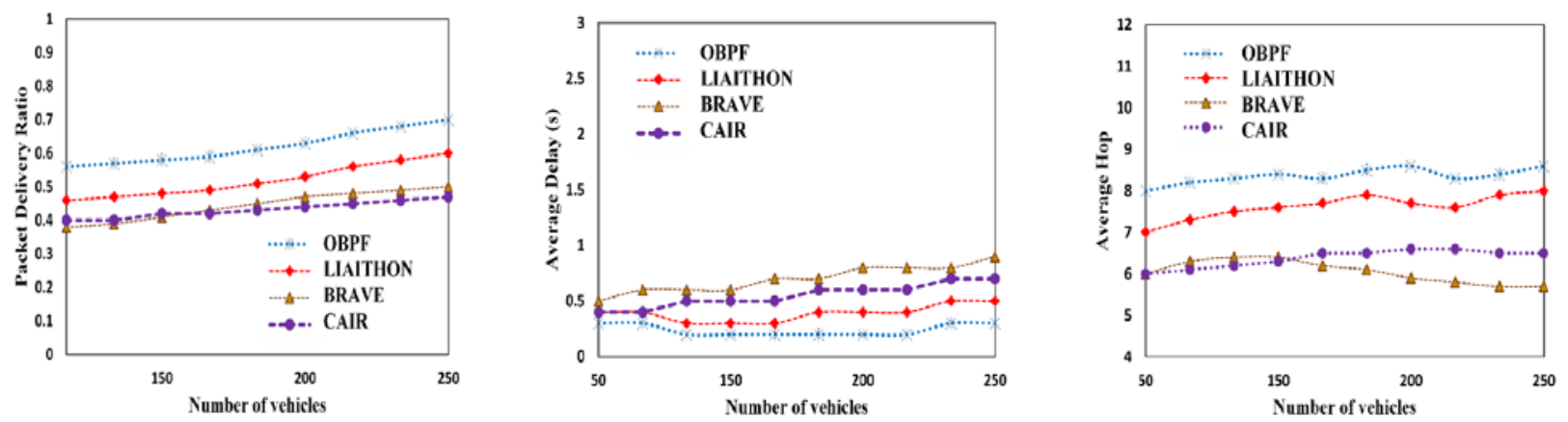

Fig. 9. Effect of traffic density on the performance of the OBPF protocol compared with LIAITHON, BRAVE and CAIR routing protocols, from left (a) packet delivery ratio (b) average delay (c) average hop count

\section{Conclusions}

In this paper, we proposed Opportunistic Beaconless Packet Forwarding Strategy for Vehicular Ad hoc Networks to optimally route the data packets toward destination. The proposed forwarding protocol is based on distributed self-election through modified 802.11 RTS/CTS frames with link quality, forward progress and directional greedy metrics. Protocols act in two modes when vehicles are between intersections and at the intersection. The protocol designed for an urban environment and considering the real traffic and realistic wireless channels. The experimental results showed that the proposed protocol performance was superior in terms of the PDR and end-to-end delay when compared with the existing LIAITHON, BRAVE and CAIR protocols. Moreover, future work involves developing a beaconless approach for highway environment, supporting data dissemination applications in VANETs [35].

\section{Acknowledgment}

The authors would like to extend their sincere appreciation to the Deanship of Scientific Research at King Saud University for funding this research. The research is supported by Ministry of Education Malaysia (MOE) and conducted in collaboration with Research Management Center (RMC) at Universiti Teknologi Malaysia (UTM) under VOT NUMBER: QJ130000.2528.06H00.

\section{References}

[1] K. N. Qureshi, A. H. Abdullah, A. Mirza, and R. W. Anwar, "Geographical Forwarding Methods in Vehicular Ad hoc Networks," International Journal of Electrical and Computer Engineering (IJECE), vol. 5, pp. 1407-1416, 2015. Article (CrossRef Link).

[2] K. N. Qureshi, A. H. Abdullah, and R. W. Anwar, "Congestion Control Scheduling Scheme for Vehicular Networks," IEEE Malaysia Section, 2014. Article (CrossRef Link).

[3] S.-H. Cha, "A survey of broadcast protocols for vehicular ad-hoc networks," SmartCR, vol. 4, pp. 246-255, 2014. Article (CrossRef Link). 
[4] K. Z. Ghafoor, M. A. Mohammed, J. Lloret, K. A. Bakar, and Z. M. Zainuddin, "Routing protocols in vehicular ad hoc networks: Survey and research challenges," Network Protocols and Algorithms, vol. 5, pp. 39-83, 2013. Article (CrossRef Link).

[5] B. Karp and H.-T. Kung, "GPSR: Greedy perimeter stateless routing for wireless networks," in Proc. of the 6th annual international conference on Mobile computing and networking, pp. 243-254, 2000. Article (CrossRef Link).

[6] C. Lochert, M. Mauve, H. Füßler, and H. Hartenstein, "Geographic routing in city scenarios," ACM SIGMOBILE Mobile Computing and Communications Review, vol. 9, pp. 69-72, 2005. Article (CrossRef Link).

[7] J. Zhao and G. Cao, "VADD: Vehicle-assisted data delivery in vehicular ad hoc networks," Vehicular Technology, IEEE Transactions on, vol. 57, pp. 1910-1922, 2008. Article (CrossRef Link).

[8] Y. Wu, S. Deng, and H. Huang, "Optimal Forwarding and Beaconing Control of Epidemic Routing in Delay Tolerant Networks," Ad-hoc \& sensor wireless networks, vol. 20, pp. 71-93, 2014. Article (CrossRef Link).

[9] T. Li, S. K. Hazra, and W. Seah, "A position-based routing protocol for metropolitan bus networks," in Proc. of Vehicular Technology Conference, 2005. VTC 2005-Spring. 2005 IEEE 61st, Stockholm, Sweden, pp. 2315-2319, 2005. Article (CrossRef Link).

[10] K. N. Qureshi, A. H. Abdullah, and G. Ullah, "Sensor based Vehicle Environment Perception Information System," in Proc. of 4 IEEE International Conference on Ubiquitous Intelligence and Computing/International Conference on Autonomic and Trusted Computing/International Conference on Scalable Computing and Communications and Its Associated Workshops, pp. 697-700, 2014. Article (CrossRef Link).

[11] H. Füßler, H. Hartenstein, M. Mauve, W. Effelsberg, and J. Widmer, "Contention-based forwarding for street scenarios," in Proc. of 1st International Workshop in Intelligent Transportation (WIT 2004), 2004. Article (CrossRef Link).

[12] P. M. Ruiz, V. Cabrera, J. A. Martinez, and F. J. Ros, "Brave: Beacon-less routing algorithm for vehicular environments," in Proc. of Mobile Adhoc and Sensor Systems (MASS), 2010 IEEE 7th International Conference on, pp. 709-714, 2010. Article (CrossRef Link).

[13] T. Aguilar, S.-J. Syue, V. Gauthier, H. Afifi, and C.-L. Wang, "CoopGeo: A beaconless geographic cross-layer protocol for cooperative wireless ad hoc networks," Wireless Communications, IEEE Transactions on, vol. 10, pp. 2554-2565, 2011. Article (CrossRef Link).

[14] K. Z. Ghafoor, J. Lloret, K. A. Bakar, A. S. Sadiq, and S. A. B. Mussa, "Beaconing approaches in vehicular ad hoc networks: a survey," Wireless personal communications, vol. 73, pp. 885-912, 2013. Article (CrossRef Link).

[15] C. Chen, Y. Jin, Q. Pei, and N. Zhang, "A connectivity-aware intersection-based routing in VANETs," EURASIP Journal on Wireless Communications and Networking, vol. 2014, pp. 1-16, 2014. Article (CrossRef Link).

[16] M. Chawla, N. Goel, K. Kalaichelvan, A. Nayak, and I. Stojmenovic, "Beaconless position based routing with guaranteed delivery for wireless ad-hoc and sensor networks," Ad-Hoc Networking, ed: Springer, 2006, pp. 61-70. Article (CrossRef Link).

[17] H. Füßler, J. Widmer, M. Käsemann, M. Mauve, and H. Hartenstein, "Contention-based forwarding for mobile ad hoc networks," Ad Hoc Networks, vol. 1, pp. 351-369, 2003. Article (CrossRef Link).

[18] J. Nzouonta, N. Rajgure, G. Wang, and C. Borcea, "VANET routing on city roads using real-time vehicular traffic information," Vehicular Technology, IEEE Transactions on, vol. 58, pp. 3609-3626, 2009. Article (CrossRef Link).

[19] S. Ruhrup, H. Kalosha, A. Nayak, and I. Stojmenovic, "Message-efficient beaconless georouting with guaranteed delivery in wireless sensor, ad hoc, and actuator networks," Networking, IEEE/ACM Transactions on, vol. 18, pp. 95-108, 2010. Article (CrossRef Link).

[20] K. Z. Ghafoor, K. Abu Bakar, J. Lloret, R. H. Khokhar, and K. C. Lee, "Intelligent beaconless geographical forwarding for urban vehicular environments," Wireless networks, vol. 19, pp. 345-362, 2013. Article (CrossRef Link). 
[21] R. Wang, C. Rezende, H. S. Ramos, R. W. Pazzi, A. Boukerche, and A. A. F. Loureiro, "LIAITHON: A location-aware multipath video streaming scheme for urban vehicular networks," in Computers and Communications (ISCC), 2012 IEEE Symposium on, Cappadocia, 2012, pp. 000436-000441. Article (CrossRef Link).

[22] C. Rezende, H. S. Ramos, R. W. Pazzi, A. Boukerche, A. C. Frery, and A. A. F. Loureiro, "Virtus: A resilient location-aware video unicast scheme for vehicular networks," in Proc. of Communications (ICC), 2012 IEEE International Conference on, pp. 698-702, 2012. Article (CrossRef Link).

[23] P. M. Ruiz, V. Cabrera, J. Martinez, and F. J. Ros, "Brave: Beacon-less routing algorithm for vehicular environments," in Proc. of Mobile Adhoc and Sensor Systems (MASS), 2010 IEEE 7th International Conference on, San Francisco, CA, pp. 709-714, 2010. Article (CrossRef Link).

[24] J.-C. Chen, "Dijkstra’s shortest path algorithm," Journal of Formalized Mathematics, vol. 15, pp. 144-157, 2003. Article (CrossRef Link).

[25] P. Dong, H. Qian, X. Wei, S. Lan, and C. Pu, "A Beacon-Less Geographic Multipath Routing Protocol for Ad Hoc Networks," Mobile Networks and Applications, vol. 18, pp. 500-512, 2013. Article (CrossRef Link).

[26] C. H. Ke, C. C. Wei, K. W. Lin, and J. W. Ding, "A smart exponential - threshold - linear backoff mechanism for IEEE 802.11 WLANs," International Journal of Communication Systems, vol. 24, pp. 1033-1048, 2011. Article (CrossRef Link).

[27] N. Baccour, A. Koubaa, M. Ben Jamâa, D. Do Rosario, H. Youssef, M. Alves, et al., "RadiaLE: A framework for designing and assessing link quality estimators in wireless sensor networks," Ad Hoc Networks, vol. 9, pp. 1165-1185, 2011. Article (CrossRef Link).

[28] M. Heissenbüttel, T. Braun, T. Bernoulli, and M. WäLchli, "BLR: beacon-less routing algorithm for mobile ad hoc networks," Computer communications, vol. 27, pp. 1076-1086, 2004. Article (CrossRef Link).

[29] K. Egoh and S. De, "A multi-criteria receiver-side relay election approach in wireless ad hoc networks," in Proc. of Military Communications Conference, 2006. MILCOM 2006. IEEE, Washington, DC, 2006, pp. 1-7. Article (CrossRef Link).

[30] S. o. U. M. SUMO, [Online].Available: . SUMO, Simulation of Urban MObility, [Online].Available: Available: Article (CrossRef Link).

[31] I. Warner De Gooijer - Cisco Systems. (2015). ArcGIS for Desktop. Article (CrossRef Link).

[32] T. Rappaport, "Mobile Communications Principales and Practice," ed: Prentice-Hall, 1996.

[33] X.-b. Wang, Y.-l. Yang, and J.-w. An, "Multi-metric routing decisions in vanet," in Proc. of Dependable, Autonomic and Secure Computing, 2009. DASC'09. Eighth IEEE International Conference on, 2009, pp. 551-556. Article (CrossRef Link).

[34] K. C. Lee, P.-C. Cheng, and M. Gerla, "GeoCross: A geographic routing protocol in the presence of loops in urban scenarios," Ad Hoc Networks, vol. 8, pp. 474-488, 2010. Article (CrossRef Link).

[35] J. Lloret, A. Canovas, A. Catalá, and M. Garcia, "Group-based protocol and mobility model for VANETs to offer internet access," Journal of Network and Computer Applications, vol. 36, pp. 1027-1038, 2013. Article (CrossRef Link). 


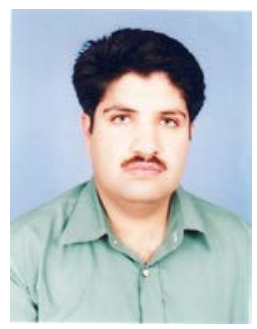

Kashif Naseer Qureshi is a Ph.D candidate at the department of Computer Systems and Communications in University of Technology Malaysia (UTM). He has received his MS Degree in Information Technology in 2012 from IM Sciences Peshawar Pakistan and MCS degree in 2003 from Baluchistan University Quetta Pakistan. He is a Cisco Certified Network Proffesional Instructor. He has eight years of experience in conducting professional trainings (CCNA, MCSE, MCITP, Web Designing, Graphic Designing) and computer wireless and wired networks and he is Microsoft (MCSE, MCITP) certified. He has been as a reviewer for various reputable academic Journals. As a part of this paper, he is working on localization based challenges in vehicular ad hoc networks applications. He has authored 25 (Scopus and ISI) journal articles, 1 book chapter and has more than 15 research papers published in national and international conferences.

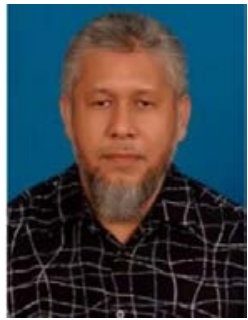

Abdul Hanan Abdullah is a Professor at University of Technology Malaysia. He obtained his Ph.D. degree from Aston University in Birmingham, United Kingdom in 1995. From 2004 to 2011, he has been the dean at the Faculty of Computer Science and Information Systems. Currently he is heading Pervasive Computing Research Group, a research group under KEconomy Research Alliances in Malaysia. His research interests include wireless sensor networks, mobile ad hoc networks, network security, internet of things, vehicular ad hoc networks and next generation networks.

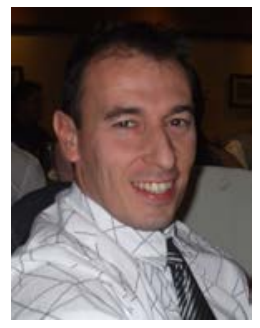

Prof. Jaime Lloret (jlloret@dcom.upv.es) received his M.Sc. in Physics in 1997, his M.Sc. in electronic Engineering in 2003 and his Ph.D. in telecommunication engineering (Dr. Ing.) in 2006. He is a Cisco Certified Network Professional Instructor. He worked as a network designer and administrator in several enterprises. He is currently Associate Professor in the Polytechnic University of Valencia. He is the head of the research group "Communications and Networks" of the Integrated Management Coastal Research Institute and he is the head of the "Active and collaborative techniques and use of technologic resources in the education (EITACURTE)" Innovation Group. He is the director of the University Diploma "Redes y Comunicaciones de Ordenadores" and the University Master "Digital Post Production". He is currently Chair of the Internet Technical Committee (IEEE Communications Society and Internet society). He has authored 22 book chapters and has more than 340 research papers published in national and international conferences, international journals (more than 110 with ISI Thomson JCR). He has been the co-editor of 38 conference proceedings and guest editor of several international books and journals. He is editor-in-chief of the "Ad Hoc and Sensor Wireless Networks" (with ISI Thomson Impact Factor), international journals "Networks Protocols and Algorithms", and International Journal of Multimedia Communications, IARIA Journals Board Chair (8 Journals) and he is (or has been) associate editor of 46 international journals (16 of them with JCR). He has been involved in more than 320 Program committees of international conferences and more than 130 organization and steering committees. He leads many national andinternational projects. He is currently the chair of the Working Group of the Standard IEEE 1907.1. He has been general chair (or co-chair) of 26 International workshops and conferences (chairman of SENSORCOMM 2007, UBICOMM 2008, ICNS 2009, ICWMC 2010, eKNOW 2012, SERVICE COMPUTATION 2013, COGNITIVE 2013, and ADAPTIVE 2013, and co-chairman of ICAS 2009, INTERNET 2010, MARSS 2011, IEEE MASS 2011, SCPA 2011, ICDS 2012, 2nd IEEE SCPA 2012, GreeNets 2012, 3rd IEEE SCPA 2013, SSPA 2013, AdHocNow 2014, MARSS 2014, SSPA 2014 IEEE CCAN 2015, 4th IEEE SCPA 2015, IEEE SCAN 2015 and local chair of MIC-WCMC 2013 and IEEE Sensors 2014). He is currently co-chair of ICACCI 2015 and SDRANCAN 2015. He is IEEE Senior and IARIA Fellow. 


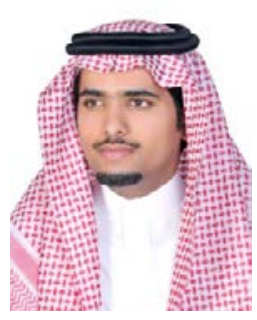

Ayman Altameem is vice dean in college of applied studies and community services King Saud University Riyadh KSA. He received his PhD in Information Technology, Computing, University of Bradford, Bradford, UK. and M.Sc in Information Systems, Computing, London South Bank University, London, UK. His keen interests are E-commerce, Internet of things, information security and artificial intelligence. 